\title{
Entanglement and sensitivity in precision measurements with states of a fluctuating number of particles
}

\author{
P. Hyllus ${ }^{1}$, L. Pezzé ${ }^{2}$ and A. Smerzi ${ }^{1}$ \\ ${ }^{1}$ INO-CNR BEC Center and Dipartimento di Fisica, Università di Trento, I-38123 Povo, Italy \\ ${ }^{2}$ Laboratoire Charles Fabry de IInstitut dOptique, CNRS and Univ. Paris-Sud, \\ Campus Polytechnique, RD 128, F-91127 Palaiseau cedex, France
}

(Dated: August 21, 2018)

\begin{abstract}
The concepts of separability, entanglement, spin-squeezing and Heisenberg limit are central in the theory of quantum enhanced metrology. In the current literature, these are well established only in the case of linear interferometers operating with input quantum states of a known fixed number of particles. This manuscript generalizes these concepts and extends the quantum phase estimation theory by taking into account classical and quantum fluctuations of the particle number. Our analysis concerns most of the current experiments on precision measurements where the number of particles is known only in average.
\end{abstract}

PACS numbers: 03.67.Bg, 06.20.Dk, 42.50.St, 42.50.Dv

Introduction. After the first pioneer works of the 80's and early 90's [1 4], the field of quantum-enhanced metrology is witnessing a revival of interest due to possible implications on fundamental questions of quantum information and technological applications. Recent theoretical analyses are mainly focusing on the interplay between spin-squeezing, entanglement and phase sensitivity [5, 6], the set of fundamental sensitivity bounds [79] and the quest for optimal phase estimation protocols [10,11]. This activity has came along with breakthrough experimental investigations with photons [12], ions [13], cold atoms [14] and Bose-Einstein condensates (BECs) 15, 16]. The interplay between theory and experiments is playing a crucial role for the development of the field. It should be noticed, however, that while the theoretical investigations have been developed in the context of systems having a fixed, known, number of particles, most experiments have been performed with a fluctuating number of particles. The consequences of classical and quantum fluctuations have been generally disregarded.

The goal of this manuscript is to extend along this direction the quantum metrology theory, and, in particular, to discuss the relation between separability, entanglement, spin-squeezing and sub shot-noise sensitivity, and to settle the concept of the Heisenberg limit. In this framework, we recognize as entangled, for instance, the state suggested by Caves in '81 in the context of MachZehnder interferometry [1], which provided the first proposal for sub shot-noise phase estimation.

Separability and Entanglement. The fluctuation of the total number of particles can have a classical and/or quantum nature. It is generally believed that quantum coherences between states of different numbers of particles do not play any observable role because of the existence of superselection rules (SSR) for the total number of particles [17-19]. Therefore, the most general states can be written as

$$
\hat{\rho}_{\mathrm{inc}}=\sum_{N=0}^{+\infty} Q_{N} \hat{\rho}^{(N)},
$$

where $\hat{\rho}^{(N)}$ is a state of $N$ particles. We extend the usual definition of separability of states of $N$ particles [20] by considering such incoherent mixtures as separable if they can be written as

$$
\hat{\rho}_{\mathrm{sep}}=\sum_{N=0}^{+\infty} Q_{N} \hat{\rho}_{\mathrm{sep}}^{(N)}
$$

where $\hat{\rho}_{\text {sep }}^{(N)}=\sum_{k} P_{k, N}\left|\phi_{k, N}^{(1)}\right\rangle\left\langle\phi_{k, N}^{(1)}|\otimes \cdots \otimes| \phi_{k, N}^{(N)}\right\rangle\left\langle\phi_{k, N}^{(N)}\right|$, $\left\{Q_{N}\right\}$ and $\left\{P_{k, N}\right\}$ are probability distributions and $\left|\phi_{k, N}^{(j)}\right\rangle$ is a two-mode pure state of a single particle [21]. The existence of a SSR is the consequence of the lack of a suitable phase reference frame (RF) 19]. However, the possibility that a suitable RF can be established in principle cannot be excluded [19]. If SSRs are lifted, then states $\hat{\rho}_{\text {coh }}$ containing coherent superpositions of different numbers of particles would become physically relevant. These will be called separable if they are separable in every fixed- $N$ subspace, i.e., if the state $\hat{\rho}_{\text {red }} \equiv \sum_{N} \mathbb{1}_{N} \hat{\rho}_{\text {coh }} \mathbb{1}_{N}$ without such coherences is of the form of Eq. (21) [21]. States which are not separable according to this definition are entangled [22]. In this manuscript, it will be shown that entanglement is a necessary resource for sub shot-noise sensitivity in linear interferometers.

Phase sensitivity with a linear interferometer. We consider a linear two-mode interferometer where the density matrix $\hat{\rho}$ evolves as $\hat{\rho}(\theta)=e^{-i \theta \hat{J}_{\vec{n}}} \hat{\rho} e^{+i \theta \hat{J}_{\vec{n}}}$, where $\theta$ is a real number, $\vec{n}$ is an arbitrary direction in the three dimensional space, and $\hat{J}_{\vec{n}}=\overrightarrow{\hat{J}} \cdot \vec{n}$ is a collective spin operator. For a non-fixed number of particles $\overrightarrow{\hat{J}}$ is defined as $\overrightarrow{\hat{J}}=\oplus_{N=1}^{+\infty} \overrightarrow{\hat{\jmath}}^{(N)}$, where $\overrightarrow{\hat{J}}^{(N)}=\frac{1}{2} \sum_{l=1}^{N} \overrightarrow{\hat{\sigma}}^{(l)}$ and $\overrightarrow{\hat{\sigma}}^{(i)}$ is the vector of Pauli matrices acting on the $l$-th particle 
24]. Note that linear interferometers preserve the number of particles, $\left[\hat{J}_{\vec{n}}, \hat{N}\right]=0$. The goal is to estimate the parameter $\theta$ with the maximum possible sensitivity $\Delta \theta$. The quantum Cramer-Rao theorem ensures that for arbitrary, unbiased, phase estimation protocols, the phase sensitivity is bounded by [25, 26]

$$
\Delta \theta_{\mathrm{QCR}}=\frac{1}{\sqrt{m F_{Q}\left[\hat{J}_{\vec{n}}, \hat{\rho}\right]}} .
$$

Here $m$ is the number of independent measurements done with identical input states $\hat{\rho}$ and $F_{Q}\left[\hat{J}_{\vec{n}}, \hat{\rho}\right]$ is the quantum Fisher information (QFI) [26]. Equation (3) is a bound on $\Delta \theta$ given the state $\hat{\rho}$ and the number of measurements $m$ and can be saturated in the central limit.

In the following, we set the fundamental sensitivity bounds of the phase estimation problem by taking, as a constraint, the average total number of particles, $\bar{N}_{\text {tot }}=$ $m\langle\hat{N}\rangle$, without fixing $m$ or $\langle\hat{N}\rangle$ separately. This correctly accounts for the finite available resources [10, 27]. Averages are computed with respect to the input state of the interferometer.

Shot-noise limit. We define the shot-noise limit as the maximal phase sensitity attainable with separable states. For separable incoherent mixtures, Eq. (2), $F_{Q}\left[\hat{\rho}_{\mathrm{sep}}, \hat{J}_{\vec{n}}\right]=\sum_{N} Q_{N} F_{Q}\left[\hat{\rho}_{\mathrm{sep}}^{(N)}, \hat{J}_{\vec{n}}^{(N)}\right] \leq \sum_{N} Q_{N} N=$ $\langle\hat{N}\rangle$. The first equality is due to the block-diagonal form of $\hat{J}_{\vec{n}}$ and $\hat{\rho}_{\text {sep }}$ [28] and the second inequality was proved in Ref. [6]. By using this result and Eq. (3), the shotnoise limit is thus given by

$$
\Delta \theta_{\mathrm{SN}}=\frac{1}{\sqrt{\overline{\bar{N}}_{\mathrm{tot}}}}
$$

which agrees with the common definition of the shotnoise or standard quantum limit. As shown in Appendix A, Eq. (4) holds also when considering separable states with coherences. This brings us to the following results.

Entanglement and sub shot-noise sensitivity. An arbitrary state with non-fixed number of particles is entangled if it fulfills the inequality

$$
\chi^{2} \equiv \frac{\langle\hat{N}\rangle}{F_{Q}\left[\hat{J}_{\vec{n}}, \hat{\rho}\right]}<1,
$$

for some direction $\vec{n}$. States satisfying Eq. (5) are useful in a linear interferometer implemented by the transformation $\hat{J}_{\vec{n}}$, since, according to Eq. (3), they provide a sub shot-noise (SSN) phase sensitvity. We further introduce the spin-squeezing condition

$$
\xi^{2} \equiv \frac{\langle\hat{N}\rangle\left(\Delta \hat{J}_{\vec{n}_{3}}\right)^{2}}{\left\langle\hat{J}_{\vec{n}_{1}}\right\rangle^{2}+\left\langle\hat{J}_{\vec{n}_{2}}\right\rangle^{2}}<1,
$$

where the vectors $\vec{n}_{1}, \vec{n}_{2}$ and $\vec{n}_{3}$ define a right-handed coordinate system [29]. In Appendix B, we prove that $\chi^{2} \leq \xi^{2}$ holds. Hence, spin-squeezed states $(\xi<1)$ are entangled and useful for SSN interferometry. Equations (5) and (6) generalize the conditions for entanglement and SSN discussed in [3, 5, , 6] to states of non-fixed number of particles and only require the replacement of $N$ with $\langle\hat{N}\rangle$. This result has been previously used without justification in several experimental works.

The Heisenberg limit. While it is well known that the shot-noise limit Eq. (4) can be overcome, finding the Heisenberg limit (HL), i.e. the ultimate scaling of the phase sensitivity imposed by Quantum Mechanics for states with non-fixed $N$, has generated a vivid debate [8, 27, 30 32]. For instance, it has been suggested that $\Delta \theta \sim 1 /\langle\hat{N}\rangle$ is the fundamental sensitivity bound 30]. Recently it was argued that the HL is given by $\Delta \theta=1 / \sqrt{\left\langle\hat{N}^{2}\right\rangle}[8]$. Not only this limit overcomes the bound $\Delta \theta=1 /\langle\hat{N}\rangle$ but, since the quantity $\left\langle\hat{N}^{2}\right\rangle$ can grow arbitrarily fast with $\langle\hat{N}\rangle$, the phase sensitivity can be arbitrarily high when considering wildly fluctuating number distributions 33.

The definition of the HL depends on the specific constraints imposed on the resources. In the presence of the SSR, and by taking $\langle\hat{N}\rangle,\left\langle\hat{N}^{2}\right\rangle$ and $m$ as constraints, the phase sensitivity is bounded by

$$
\Delta \theta \geq \max \left[\frac{1}{\sqrt{m\left\langle\hat{N}^{2}\right\rangle}}, \frac{1}{m\langle\hat{N}\rangle}\right] .
$$

The first bound is derived from Eq. (3) by noticing that, for incoherent mixtures (1), $F_{Q}\left[\hat{\rho}_{\text {inc }}, \hat{J}_{\vec{n}}\right]=$ $\sum_{N} Q_{N} F_{Q}\left[\hat{\rho}^{(N)}, \hat{J}_{\vec{n}}^{(N)}\right] \leq \sum_{N} Q_{N} N^{2}=\left\langle\hat{N}^{2}\right\rangle$. It agrees with the bound of [8], except for the factor $m$, accounting for repeated independent measurements. Note that in the central limit, the bound scales as $1 / \sqrt{m}$. The second bound is proven in Appendix C. When the number of particles is fixed and equal to $N$, Eq. (7) recovers the definition of the HL $\Delta \theta=1 / \sqrt{m} N$ discussed in [7] which takes, as constraints, $N$ and $m$, separately. Note, however, that from this limit one does not obtain Eq. (17) by naively replacing $N \rightarrow\langle\hat{N}\rangle$.

Here, we define the HL as the maximum phase sensitivity attainable with fixed $\bar{N}_{\text {tot }}$. If coherences can neither be generated nor measured, as in current experimental interferometric protocols because of the SSR, the HL is

$$
\Delta \theta_{\mathrm{HL}}=\frac{1}{\bar{N}_{\text {tot }}}
$$

If the state contains coherences but only POVMs without number-coherences are applied, the HL is still given by Eq. (8), cf. Appendix C. In particular, this is true for the Mach-Zehnder interferometer, even if only the relative number of particles is measured or the parity at one exit port. The latter measurement was considered in Ref. [31]. By adapting the proof in Appendix $\mathrm{C}$ it can also be shown that the HL Eq. (8) holds for incoherent mixtures even if POVMs with coherences are available. 
If the state contains coherences and POVMs with coherences are used, then Eq. (8) is valid in the central limit only [34]. Outside the central limit the bound

$$
\Delta \theta \geq \frac{1}{\sqrt{m\left\langle\hat{N}^{2}\right\rangle}}
$$

holds, as shown in Appendix C. However, there are arguments suggesting that the HL may be given by Eq. (8) also in this case 27, 35.

Examples. We consider a Mach-Zehnder interferometer, corresponding to a collective rotation around the $y$ axis, i.e., $\vec{n}=\hat{y}$, with a coherent state $|\alpha\rangle_{a}$ in the input mode $a$ and a squeezed state $|\zeta\rangle_{b}$ in the input $b$ as suggested by Caves [1, 10, 36]. Here $\alpha=|\alpha| e^{i \phi_{\alpha}}$ and $\zeta=r e^{i \theta_{\zeta}}$. When fixing the relative phase $2 \phi_{\alpha}=\theta$, Eq. (5) gives

$$
\chi^{2}=\frac{|\alpha|^{2}+\sinh ^{2} r}{|\alpha|^{2} e^{2 r}+\sinh ^{2} r} .
$$

For $r=0$ we have a coherent state entering one of the two ports and vacuum the other port. We obtain $\chi^{2}=$ 1 and hence shot noise, as expected. In contrast, we obtain $\chi^{2}<1$ when $r>0$ and $|\alpha|^{2} \neq 0$. The state is entangled and, for relatively small values of $r$, it is also spin squeezed, according to the definition Eq. (6). A direct calculation for $\vec{n}_{3}=\hat{x}$ gives

$$
\xi^{2}=\frac{\left(|\alpha|^{2}+\sinh ^{2} r\right)\left(|\alpha|^{2} e^{-2 r}+\sinh ^{2} r\right)}{\left(|\alpha|^{2}-\sinh ^{2} r\right)^{2}} .
$$

In the limit $|\alpha|^{2} \gg \sinh ^{2} r$ we have that $\xi^{2}=e^{-2 r}<$ 1 for $r>0$ (and $|\alpha| \neq 0$ ). Spin squeezing is lost for $|\alpha|^{2} \sim \sinh ^{2} r$, even though the state remains entangled. Eqs (1011) hold even for the state $\rho_{\text {red }}=$ $\sum_{N} \mathbb{1}_{N}|\alpha, \zeta\rangle\langle\alpha, \zeta| \mathbb{1}_{N}$ obtained in the presence of the SSR [19, 37]. We finally notice that the state $|\alpha\rangle \otimes|\zeta\rangle$ reaches the HL $\Delta \theta \sim 1 / \bar{N}_{\text {tot }}$ when $|\alpha|^{2} \approx \sinh ^{2} r[10$.

Conclusions. In this manuscript we have extended the quantum phase estimation theory to the case of states with fluctuating number of particles. In particular, we have discussed entanglement and spin-squeezing and provided the class of entangled states useful for sub shotnoise sensitivity. The Heisenberg limit has been defined by taking into account the finite available resources. Our results are relevant for most current experiments on the creation, detection and application of entangled states with a fluctuating number of particles for quantum metrology.

Appendix A. Shot-noise limit. Here we demonstrate that $F_{Q}\left(\hat{\rho}_{\mathrm{cs}}\right) \leq\langle\hat{N}\rangle$ for states of the form $\hat{\rho}_{\mathrm{cs}}=\sum_{k} p_{k}\left|\psi_{\mathrm{cs}}^{(k)}\right\rangle\left\langle\psi_{\mathrm{cs}}^{(k)}\right|$, which contain coherences between different numbers of particles and are separable in every fixed- $N$ subspace. We obtain $F_{Q}\left(\hat{\rho}_{\mathrm{cs}}\right) \leq$ $\max 4 \sum_{k} p_{k}\left(\Delta \hat{J}_{\vec{n}}\right)_{\left|\psi_{\mathrm{cs}}^{(k)}\right\rangle}^{2} \leq 4 \sum_{k} p_{k} \sum_{N} Q_{N}^{(k)} \max$
$\left\langle\left[\hat{J}_{\vec{n}}^{(N)}\right]^{2}\right\rangle_{\left|\psi_{\mathrm{cs}}^{(N, k)}\right\rangle} \leq \sum_{k} p_{k} \sum_{N} Q_{N}^{(k)} N=\langle\hat{N}\rangle$, by using the convexity of the quantum Fisher information [38]. We expanded $\left|\psi_{\mathrm{cs}}^{(k)}\right\rangle=\sum_{N} \sqrt{Q_{N}^{(k)}}\left|\psi_{\mathrm{cs}}^{(N, k)}\right\rangle$ and used that for pure separable states $\left\langle\left[\hat{J}_{\text {vec }}^{(N)}\right]^{2}\right\rangle_{\left|\psi_{\text {sep }}^{(N)}\right\rangle} \leq \frac{N}{4}[\underline{6}]$. By inserting $F_{Q}\left(\hat{\rho}_{\mathrm{cS}}\right) \leq\langle\hat{N}\rangle$ into Eq. (3), we recover Eq. (44).

Appendix B. Spin-squeezing inequality. We consider, without loss of generality, a coordinate system such that $\left\langle\hat{J}_{\vec{n}_{2}}\right\rangle=0$. From the inequalities $F_{Q}\left[\hat{\rho}, \hat{J}_{\vec{n}}\right] \geq F_{\hat{E}(\varepsilon)}[\hat{\rho}(\theta)]$ [26] and $F_{\hat{E}(\varepsilon)}[\hat{\rho}(\theta)] \geq \frac{1}{(\Delta \hat{A})^{2}}\left(\frac{\mathrm{d}\langle\hat{A}\rangle}{\mathrm{d} \theta}\right)^{2}$ (obtained by extending the proof in 39] to the case of non-fixed number of particles), it follows that $\chi^{2} \leq\langle\hat{N}\rangle(\Delta \hat{A})^{2} /(\mathrm{d}\langle\hat{A}\rangle / \mathrm{d} \theta)^{2}$. By choosing $\vec{n}=\vec{n}_{2}, \hat{A}=\hat{J}_{\vec{n}_{3}}$ and using the commutation relations of the $\hat{J}_{\vec{n}_{i}}$ operators [29], we obtain $\frac{\partial}{\partial \theta}\left\langle\hat{J}_{\vec{n}_{3}}\right\rangle=$ $i \operatorname{Tr}\left[\hat{J}_{\vec{n}_{3}}\left[\hat{J}_{\vec{n}_{2}}, \hat{\rho}(\theta)\right]\right]=\left\langle\hat{J}_{\vec{n}_{1}}\right\rangle$. Hence $\chi^{2} \leq \frac{\left(\Delta \hat{J}_{\vec{n}_{3}}\right)^{2}}{\left\langle\hat{J}_{\vec{n}_{1}}\right\rangle^{2}}=\xi^{2}$.

Appendix C. Heisenberg limit. In the presence of the SSR, only POVMs without coherences are available. A POVM is of this form if we can write its elements as $\hat{E}(\lambda)=\sum_{(N, M) \in I(\lambda)} \hat{E}_{N, M}$, where $\sum_{M} \hat{E}_{N, M}=\mathbb{1}_{N}$ ( $M$ is a degree of freedom in each $N$-subspace), and $I(\lambda)$ are all pairs $(N, M)$ leading to the same $\lambda$ ( $\lambda$ could be the number of particles at one port of a Mach-Zehnder interferometer, for instance). For $m=1$, the conditional probabilities are $P(\lambda)=$ $\sum_{(N, M) \in I(\lambda)} P(N, M \mid \theta)=\sum_{(N, M) \in I(\lambda)} Q_{N} P(M \mid \theta, N)$, where $P(M \mid \theta, N)=\operatorname{Tr}\left[\hat{E}_{N, M} e^{i \theta \hat{J}_{\vec{n}}} \hat{\rho}^{(N)} e^{-i \theta \hat{J}_{\vec{n}}}\right]$, and $Q_{N} \hat{\rho}^{(N)}=\mathbb{1}_{N} \hat{\rho} \mathbb{1}_{N}$. The variance of an estimator $\theta_{\text {est }}(\lambda)$ (assumed to be unbiased, i.e., $\left.\bar{\theta}_{\text {est }}=\theta\right)$ is $\left(\Delta \theta_{\text {est }}\right)^{2}=\sum_{\lambda} P(\lambda \mid \theta)\left(\theta_{\text {est }}(\lambda)-\theta\right)^{2}=$ $\sum_{\lambda} \sum_{(N, M) \in I(\lambda)} Q_{N} P(M \mid \theta, N)\left(\theta_{\text {est }}(\lambda)-\theta\right)^{2}=$ $\sum_{N} Q_{N} \sum_{M} P(M \mid N, \theta)\left(\theta_{\text {est }}(\lambda[N, M])-\theta\right)^{2}=$ $\sum_{N} Q_{N}\left(\Delta \theta_{\text {est }}^{(N)}\right)^{2} \geq \sum_{N} Q_{N} / N^{2}$. We used that $\sum_{\lambda} \sum_{(N, M) \in I(\lambda)}=\sum_{N} \sum_{M}$ holds since $\sum_{\lambda} \hat{E}(\lambda)=\mathbb{1}$. In the last inequality we used that $\left(\Delta \theta_{\text {est }}^{(\vec{N})}\right)^{2} \geq 1 / N^{2}$ holds for unbiased estimators [7]. For $m \geq 1$, $P(\vec{\lambda} \mid \theta)=\prod_{i=1}^{m} P\left(\lambda_{i} \mid \theta\right)$ and it can be shown that $\left(\Delta \theta_{\text {est }}\right)^{2} \geq \sum_{\vec{N}} Q_{\vec{N}} / \sum_{i=1}^{m} N_{i}^{2} \geq \sum_{\vec{N}} Q_{\vec{N}} /\left(\sum_{i=1}^{m} N_{i}\right)^{2}$, where the first inequality follows as above and the second inequality holds for positive numbers. For $m=1$, we then obtain $\left(\sum_{N} Q_{N} / N^{2}\right)\left(\sum_{N} Q_{N}\right) \geq\left(\sum_{N} Q_{N} / N\right)^{2}$ from the Cauchy-Schwartz inequality. Applying it again leads to $\left(\sum_{N} Q_{N} / N\right)\left(\sum_{N} Q_{N} N\right) \geq\left(\sum_{N} Q_{N}\right)^{2}=1$. Therefore, we arrive at $\Delta \theta_{\text {est }} \geq 1 /\langle\hat{N}\rangle$. This can be done in analogy for $m \geq 1$. This proves that $\Delta \theta_{\text {est }} \geq 1 / m\langle\hat{N}\rangle$.

The bound (9) can be proven as in Appendix A by using general states and $F_{Q}\left[\left|\psi^{(N)}\right\rangle, \hat{J}_{\vec{n}}\right] \leq N^{2}$.

Acknowledgements. We thank J.I. Cirac, K. Mølmer, A.S. Sørensen and T. Rudolph for stimulating discussions. 
[1] C.M. Caves, Phys. Rev. D 23, 1693 (1981).

[2] B. Yurke, S.L. McCall and J.R. Klauder, Phys. Rev. A 33, 4033 (1986); M. Xiao, L.A. Wu and H.J. Kimble, Phys. Rev. Lett. 59, 278 (1987); P. Grangier, R.E. Slusher, B. Yurke and A. LaPorta, Phys. Rev. Lett. 59, 2153 (1987); M.J. Holland and K. Burnett, Phys. Rev. Lett. 71, 1355 (1993).

[3] D.J. Wineland, J.J. Bollinger, W.M. Itano, and D.J. Heinzen, Phys. Rev. A 50, 67 (1994).

[4] M. Kitagawa and M. Ueda, Phys. Rev. A 47, 5138 (1993).

[5] A. Sørensen, et al., Nature 409, 63 (2001).

[6] L. Pezzé and A. Smerzi, Phys. Rev. Lett. 102, 100401 (2009).

[7] V. Giovannetti, S. Lloyd and L. Maccone, Phys. Rev. Lett. 96, 010401 (2006).

[8] H.F. Hofmann, Phys. Rev. A 79, 033822 (2009).

[9] Á. Rivas and A. Luis, Phys. Rev. Lett. 105, 010403 (2010).

[10] L. Pezzé and A. Smerzi, Phys. Rev. Lett. 100, 073601 (2008).

[11] Y.P. Huang and M.G. Moore, Phys. Rev. Lett. 100, 250406 (2008); U. Dorner, et al., Phys. Rev. Lett. 102, 040403 (2009); D.W. Berry, et al., Phys. Rev. A 80, 052114 (2009);

[12] P. Walther, et al., Nature 429, 158 (2004); M.W. Mitchell, et al., Nature 429, 161 (2004); T. Nagata, et al., Science 316, 726 (2007).

[13] V. Meyer et al., Phys. Rev. Lett. 86, 5870 (2001); D. Leibfried et al., Science 304, 1476 (2004).

[14] J. Appel, et al., PNAS 106, 10960 (2009); M.H. SchleierSmith, I.D. Leroux, V. Vuletic, Phys. Rev. Lett. 104, 073604 (2010).

[15] G.B. Jo et al., Phys. Rev. Lett. 98, 030407 (2007); J. Estève, et al., Nature 455, 1216 (2008).

[16] P. Böhi, et al., Nat. Phys 5, 592 (2009).

[17] G.C. Wick, et al., Phys. Rev. 88, 101 (1952).

[18] B.C. Sanders, S.D. Bartlett, T. Rudolph and P.L. Knight, Phys. Rev. A 68, 042329 (2003); K. Mølmer, Phys. Rev. A 55, 3195 (1997);

[19] S.D. Bartlett et al. Rev. Mod. Phys. 79, 555 (2007).

[20] R.F. Werner, Phys. Rev. A 40, 4277 (1989).

[21] Density matrices $\hat{\rho}^{(N)}$ of $N$ particles with 2 degrees of freedom act on a Hilbert space $\mathcal{H}_{N}=\otimes_{j=1}^{N} \mathcal{H}_{N}^{(j)}$, where $\mathcal{H}_{N}^{(j)} \simeq \mathbb{C}^{2}$. The operator $\mathbb{1}_{N}$ is the projector onto $\mathcal{H}_{N}$.

[22] The definition of separability can also be applied formally when treating indistinguishable particles in first quantization, where the set of admissible quantum states is reduced to the symmetric/antisymmetric subspace for bosons/fermions. Separable states of bosons without coherences are then given by Eq. (2) with $\left|\phi_{k, N}^{(i)}\right\rangle=\left|\phi_{k, N}\right\rangle$ for all $i$. It has been argued that particle entanglement due to (anti-)symmetrization in first quantization is unphysical since the individual particles cannot be addressed 23]. However, in interferometers using only collective operations, it can still be a useful resource for sub shot-noise sensitivity.

[23] P. Zanardi, Phys. Rev. A 65, 042101 (2002); A. P. Hines, R. H. McKenzie, and G. J. Milburn, Phys. Rev. A 67, 013609 (2003); F. Benatti, R. Floreanini, and U. Marzolino, Ann. Phys. 325, 924 (2010). See also L. Amico et al., Rev. Mod. Phys. 80, 517 (2008) and References within.

[24] For bosons, the operators $\hat{J}_{x, y, z}$ can be written as $\hat{J}_{x}=$ $\left(\hat{a}^{\dagger} \hat{b}+\hat{b}^{\dagger} \hat{a}\right) / 2, \hat{J}_{y}=\left(\hat{a}^{\dagger} \hat{b}-\hat{b}^{\dagger} \hat{a}\right) / 2 i$, and $\hat{J}_{z}=\left(\hat{a}^{\dagger} \hat{a}-\right.$ $\left.\hat{b}^{\dagger} \hat{b}\right) / 2$ using annihilation operators $\hat{a}$ and $\hat{b}$ for the two interferometric modes.

[25] S.L. Braunstein and C.M. Caves, Phys. Rev. Lett. 72, 3439 (1994).

[26] To estimate the parameter $\theta$, a positive operator value measurement (POVM), $\{\hat{E}(\varepsilon)\}$, is performed on the output state and $\theta$ is inferred from the result of this measurement. The maximum sensitivity of this estimation is bounded by the Cramer-Rao bound $\Delta \theta_{\mathrm{CR}}=$ $1 / \sqrt{m F_{\hat{E}(\varepsilon)}[\hat{\rho}(\theta)]}$, where $m$ is the number of measurements, $F_{\hat{E}(\varepsilon)}[\hat{\rho}(\theta)]=\int d \varepsilon P(\varepsilon \mid \theta)\left(\partial_{\theta} \log P(\varepsilon \mid \theta) / \partial \theta\right)^{2}$ is the Fisher information and $P(\varepsilon \mid \theta)=\operatorname{Tr}[\hat{E}(\varepsilon) \hat{\rho}(\theta)]$. The QFI is the maximum $F_{Q}[\hat{\rho}(\theta)] \equiv \max _{\{\hat{E}(\varepsilon)\}} F_{\hat{E}(\varepsilon)}[\hat{\rho}(\theta)]$ and it is saturated by a particular POVM 25].

[27] S.L. Braunstein, A.S. Lane and C.M. Caves, Phys. Rev. Lett. 69, 2153 (1992); A.S. Lane, S.L. Braunstein and C.M. Caves, Phys. Rev. A 47, 1667 (1993).

[28] The QFI for a state of fixed number of particles, $\hat{\rho}^{(N)}$, is $F_{\mathrm{Q}}\left[\hat{\rho}^{(N)}, \hat{J}_{\vec{n}}^{(N)}\right]=4\left(\Delta \hat{R}^{(N)}\right)^{2}$, where the Hermitean operator $\hat{R}^{(N)}$ satisfies the equation $\left\{\hat{R}^{(N)}, \hat{\rho}^{(N)}\right\}=$ $i\left[\hat{J}_{\vec{n}}^{(N)}, \hat{\rho}^{(N)}\right]$. For pure states, $F_{Q}\left[|\psi\rangle, \hat{J}_{\vec{n}}\right]=4\left(\Delta \hat{J}_{\vec{n}}\right)_{|\psi\rangle}^{2}$. If the input state is an incoherent mixture $\hat{\rho}=\sum_{N} Q_{N} \hat{\rho}^{(N)}$, then $F_{\mathrm{Q}}\left[\hat{\rho}, \hat{J}_{\vec{n}}\right]=\sum_{N} Q_{N} F_{\mathrm{Q}}\left[\hat{\rho}^{(N)}, \hat{J}_{\vec{n}}^{(N)}\right]$ follows since $\hat{\rho}$ and $\hat{J}_{\vec{n}}$ commute with $\hat{N}$, which can also be imposed for $\hat{R}$. The POVM saturating $F=F_{Q}$ can be chosen as a von Neumann measurement on the eigenstates of $\hat{R}$ 25], therefore a POVM without coherences saturates $F_{Q}$. However, for non-restricted states and POVMs $F_{Q}(\hat{\rho}) \geq F_{Q}\left(\sum_{N} \mathbb{1}_{N} \hat{\rho} \mathbb{1}_{N}\right)$ holds. A simple example where the inequality is strict is $(|y+\rangle+|y-\rangle \otimes|z+\rangle) / \sqrt{2}$ for the generator $\hat{J}_{y}$, where $|(y, z) \pm\rangle$ are the eigenstates of $\hat{\sigma}_{y, z}$.

[29] For a right-handed coordinate system $\vec{n}_{i}, i=1,2,3$, the operators $\hat{J}_{\vec{n}_{i}}$ satisfy the $\mathrm{SU}(2)$ algebra of angular momentum: $\left[\hat{J}_{\vec{n}_{i}}, \hat{J}_{\vec{n}_{j}}\right]=i \epsilon_{i, j, k} \hat{J}_{\vec{n}_{k}}$, where $\epsilon_{i, j, k}$ is the LeviCivita tensor.

[30] Z.Y. Ou, Phys. Rev. A 55, 2598 (1997).

[31] P.M. Anisimov, et al., Phys. Rev. Lett. 104, 103602 (2010).

[32] J.H. Shapiro, S.R. Shepard and N.C. Wong, Phys. Rev. Lett. 62, 2377 (1989).

[33] This happens, for instance, for the state proposed by Shapiro, Shepard and Wong (SSW) 32]. However, the SSW result did not take into account the number of measurements. Indeed, it was shown that the SSW proposal does not overcome the limit $\Delta \theta=1 / \bar{N}_{\text {tot }}[27]$.

[34] Fisher's theorem ensures that the bound $1 /\left(m\left\langle\hat{N}^{2}\right\rangle\right)^{1 / 2}$ can be saturated in the central limit for $m \geq m_{\mathrm{cl}}$, where $m_{\mathrm{cl}}$ depends on the POVM used. In particular, it can be saturated with POVMs without coherences. In this case, and for $m \geq m_{\mathrm{cl}}^{\text {inc }}, 1 /\left(m\left\langle\hat{N}^{2}\right\rangle\right)^{1 / 2} \geq 1 / m\langle\hat{N}\rangle$ holds due to Eq. (7). However, for $m^{\prime}<m_{\mathrm{cl}}^{\text {inc }}$, we cannot rule out that $\Delta \theta<1 / \bar{N}_{\text {tot }}$ can be achieved if states and POVMs with coherences are available.

[35] L.B. Levitin and T. Toffoli, Phys. Rev. Lett. 103, 160502 (2009).

[36] The state $|\alpha\rangle \otimes|\zeta\rangle$ ( $\otimes$ is the mode tensor product here) 
reaches a SSN sensitivity $\Delta \theta=\epsilon / \sqrt{|\alpha|^{2}}$, with $\epsilon<1[1]$.

[37] For the considered states written as $|\psi\rangle \equiv$ $\sum_{N} \sqrt{Q_{N}}\left|\psi^{(N)}\right\rangle$, one can show that $\left\langle\hat{J}_{x, y}^{(N)}\right\rangle_{\left|\psi^{(N)}\right\rangle}=0$ holds for all $N$. Eqs (1011) are invariant since $F_{Q}(|\psi\rangle)=$ $4\left(\Delta \hat{J}_{y}\right)_{|\psi\rangle}^{2}=F_{Q}\left(\hat{\rho}_{\text {red }}\right)=\sum_{N} Q_{N}\left(\Delta \hat{J}_{y}^{(N)}\right)_{|\psi(N)\rangle}^{2}$ [28] holds and for block-diagonal operators $\langle\hat{A}\rangle_{|\psi\rangle}=\langle\hat{A}\rangle_{\hat{\rho}_{\text {red }}}$.

[38] Convexity of $F$ has been proven in M. L. Cohen, IEEE Transactions on Information Theory 14, 591 (1968). A slightly more compact proof is reported here. For $\hat{\rho}(\theta)=\sum_{k} p_{k} \hat{\rho}_{k}(\theta)$ we have $P(\varepsilon \mid \theta)=\operatorname{Tr}[\hat{E}(\varepsilon) \hat{\rho}(\theta)]=$ $\sum_{k} p_{k} P_{k}(\varepsilon \mid \theta)$ where $P_{k}(\varepsilon \mid \theta)=\operatorname{Tr}\left[\hat{E}(\varepsilon) \hat{\rho}_{k}(\theta)\right]$. Using the Cauchy-Schwarz inequality we have that $(\partial P(\varepsilon \mid \theta) / \partial \theta)^{2} / P(\varepsilon \mid \theta) \leq \sum_{k} p_{k}\left(\partial P_{k}(\varepsilon \mid \theta) / \partial \theta\right)^{2} / P_{k}(\varepsilon \mid \theta)$. Integrating over $\mathrm{d} \varepsilon$ we obtain that $F_{\hat{E}(\varepsilon)}\left[\sum_{k} p_{k} \hat{\rho}_{k}(\theta)\right] \leq$ $\sum_{k} p_{k} F_{\hat{E}(\varepsilon)}\left[\hat{\rho}_{k}(\theta)\right]$. This inequality holds for all possible POVM and, in particular, for those saturating the QFI. [39] H. Uys and P. Meystre, Phys. Rev. A 76, 013804 (2007). 